\title{
EDUCACIÓN PARA LA CIUDADANÍA: EL GATO YA TIENE SU CASCABEL
}

\author{
Francisco Rodríguez Lestegás \\ Universidad de Santiago de Compostela
}

\section{RESUMEN}

En este trabajo se plantean las dificultades que presenta la incorporación de la educación para la ciudadanía como nueva área o materia de la educación primaria y secundaria en el sistema educativo español. Bien por atribuírsele un carácter propagandístico, por considerarla una alternativa laica a la enseñanza de la religión, por estimar más apropiado un tratamiento transversal o por entender que sus contenidos son propios de otras materias ya presentes en el currículo escolar, lo cierto es que existen serios inconvenientes para que la educación para la ciudadanía reúna los requisitos propios de un saber integrante de la cultura escolar.

PALABRAS CLAVE: educación para la ciudadanía, cultura escolar, modelo disciplinar.

\section{ABSTRACT}

This paper deals with the difficulties entailed by the incorporation of Citizenship Education as a new area or subject in primary and secondary education in the Spanish educational system. Either for attributing it a propagandistic character, for considering it a secular alternative to the teaching Religion, for regarding a cross-curriculum as more appropriate or for understanding that their contents are already included in other subjects of the school curriculum, the truth is that serious objections exist for Citizenship Education to gather the requirements of an integrating knowledge of the school culture.

KEYWORDS: citizenship education, school culture, disciplinary model.

Uno de los aspectos más novedosos, y también más polémicos, de la Ley Orgánica de Educación (LOE) se sitúa en la incorporación al currículo de la educación para la ciudadanía, con diferentes denominaciones en función de la naturaleza de los contenidos propuestos y de las edades de los alumnos a quienes va dirigida, 
desde la educación primaria hasta el bachillerato ${ }^{111}$. En el preámbulo de la mencionada disposición se apunta la finalidad de "ofrecer a todos los estudiantes un espacio de reflexión, análisis y estudio acerca de las características fundamentales y el funcionamiento de un régimen democrático, de los principios y derechos establecidos en la Constitución española y en los tratados y las declaraciones universales de los derechos humanos, así como de los valores comunes que constituyen el sustrato de la ciudadanía democrática en un contexto global".

La expresión "educación para la ciudadanía" se nos presenta con un carácter polisémico, ambiguo y de perfiles borrosos, en función de lo que previamente se entienda por "ciudadanía" y teniendo en cuenta el proyecto de sociedad al que trata de servir, los ámbitos en los que se ejerza y el enfoque bajo el que se imparta (Marco, 2002). La educación para la ciudadanía es un dominio muy oportuno para estudiar la relación entre saberes y experiencias, al tiempo que reclama convicciones y compromisos personales en mayor medida que cualquier otra parcela educativa. Así lo aprecia F. Audigier cuando, desde la consideración de la ciudadanía democrática como una cultura conflictiva, se refiere a esta dimensión formativa como "un campo teórico y práctico donde se traducen, en acuerdos y desacuerdos, nuestras concepciones de la vida social y política, la definición de los derechos, libertades y obligaciones que estimamos legítimos para nosotros y para los demás, nuestras maneras de pensar el lugar de los conflictos y de resolverlos, nuestras concepciones de la educación, del papel respectivo de las familias, de la escuela y de otras instituciones susceptibles de intervenir en la instrucción, la educación y la socialización, del lugar que ocupan los conocimientos o la experiencia en la construcción de competencias" (Audigier, 1999, p. 6).

\section{La educación para la ciudadanía goza de reconocimiento internacional}

La idea de introducir la educación para la ciudadanía en los currículos escolares de nuestro país no se debe a una más o menos oportunista ocurrencia de los expertos y políticos que han redactado y aprobado la LOE. Por el contrario, en los últimos años han venido proliferando las iniciativas de las más prestigiosas organizaciones e instituciones internacionales (UNESCO, Consejo de Europa, Asociación Internacional para la Evaluación del Rendimiento Educativo y la propia Unión Europea) en el sentido de impulsar este ámbito educativo, así como promover la enseñanza y la investigación en el mismo. De hecho, y aunque en la práctica admita una gran diversidad de configuraciones, la educación para la ciudadanía no sólo está presente en todos los sistemas educativos de los países democráticos, sino que incluso trata de abrirse paso en aquellos lugares donde las instituciones y la cultura democrática se encuentran aún en vías de consolidación (Audigier, 1999).

En concreto, desde la organización supranacional europea se entiende que "se hace cada vez más importante explicar a los jóvenes el significado de la ciudadanía responsable en una sociedad democrática, siendo igualmente necesario enseñarles los principios de una actitud cívica positiva. Por el bien de la cohesión social en Europa y de una identidad europea común, los alumnos, en los centros docentes,

[1] "Educación para la ciudadanía y los derechos humanos" en uno de los cursos del tercer ciclo de la educación primaria y en uno de los tres primeros cursos de la educación secundaria obligatoria, "Educación ético-cívica" en el cuarto curso de la educación secundaria obligatoria, y "Filosofía y ciudadanía" en el bachillerato. 
deben recibir información específica sobre el significado de la ciudadanía, los tipos de derechos y deberes que ésta conlleva, y sobre qué hacer para portarse como un "buen ciudadano" (Eurydice, 2005, p. 8).

Al margen del resabio tecnicista que puede desprenderse de la expresión entrecomillada final de esta declaración, la educación para la ciudadanía en el contexto europeo se construye sobre el concepto de "ciudadanía responsable" ${ }^{[2]}$ y hace referencia a la educación escolar que reciben niños y jóvenes, con el fin de convertirlos en ciudadanos activos y responsables, capaces de contribuir al desarrollo y al bienestar de la sociedad a la que pertenecen. Con esta intención, la educación para la ciudadanía forma parte del currículo de todos los países de la Unión Europea, aunque su organización puede diferir bastante de un país a otro. En unos casos se oferta como materia independiente, con carácter obligatorio u optativo; otras veces aparece integrada en una o más materias, como por ejemplo la historia, la geografía, los estudios sociales, la ética, la filosofía u otras; y una tercera posibilidad es la de impartirla como tema transversal, de modo que los principios en los que se apoya estén presentes en todas las materias del currículo. Además, estos distintos enfoques no se excluyen entre sí, de manera que en un mismo país pueden coexistir varias de las modalidades apuntadas.

A pesar de que los objetivos y contenidos de la educación para la ciudadanía se formulan de manera muy distinta según el país de que se trate, las propuestas educativas suelen coincidir en tratar de orientar a los estudiantes en tres direcciones: adquirir una cultura política (conocimientos teóricos sobre los derechos humanos y la democracia, funcionamiento de las instituciones políticas y sociales, etc.), desarrollar el pensamiento crítico y ciertas actitudes y valores necesarios para convertirse en ciudadanos responsables (aprender a respetarse y a respetar a los demás, saber escuchar y resolver conflictos de manera pacífica, desarrollar valores acordes con una sociedad plural, etc.), y estimular la participación activa de los alumnos con el fin de capacitarlos para implicarse, de forma constructiva y crítica, en la vida de la comunidad escolar y local (Eurydice, 2005).

\section{La educación para la ciudadanía requiere la superación de ciertos prejuicios}

Aprovechando la tribuna de un influyente medio de comunicación, la voz autorizada de Peces-Barba (2004) expresaba con determinación la necesidad de concretar la educación en valores ${ }^{[3]}$ en una materia escolar "que no puede ser improvisada, ni coyuntural, ni oportunista, sino sistemática, completa y adecuada a la edad de los

[2] Aunque esta expresión puede tener connotaciones diferentes en función del país y contexto en el que se use, es posible afirmar que, por lo general, hablar de "ciudadanía responsable" supone referirse a aspectos relacionados con el conocimiento y el ejercicio de los derechos y responsabilidades cívicas. Igualmente, el concepto se asocia siempre con valores tales como la democracia, la libertad, la igualdad, la dignidad humana, el respeto por los derechos humanos, la tolerancia, el respeto a la ley, la justicia social, la solidaridad, etc. A la concepción del ciudadano como sujeto de derechos y deberes, la ciudadanía responsable añade la consideración del mismo como individuo que participa activamente en las instancias donde se genera la política.

[3] La expresión "educación en valores" coincidía con una de las propuestas contenidas en el documento Una educación de calidad para todos y entre todos, divulgado por el Ministerio de Educación y Ciencia en septiembre de 2004 con el propósito de estimular el debate social sobre la educación antes de promover la iniciativa legislativa que conduciría a la LOE. 
alumnos y que exige una estabilidad y una permanencia para que pueda producir frutos". Pero, a continuación, señalaba la obligación de despejar ciertos prejuicios ${ }^{[4]}$ que alimentan las argumentaciones de los detractores de esta materia. Su presunto carácter sectario y propagandístico, como si la educación para la ciudadanía no fuese más que una burda reencarnación de la denostada "Formación del Espíritu Nacional" de tiempos pasados, es el primero de ellos. Quienes se sitúan en una posición de mayor beligerancia frente a esta asignatura piensan que nos han endosado una nueva educación política, una iniciativa sectaria de dirigismo ideológico-político con la que el gobierno pretende adoctrinar a los estudiantes para asegurarse la continuidad; por consiguiente, el trabajo de los profesores encargados de tal adoctrinamiento sólo tiene sentido si se presupone su adscripción ideológica a la corriente partidista gubernamental (Robles, 2005). Al margen de que esta objeción no parece muy consistente (el profesorado será el que ya existe en los centros escolares, por lo que su pluralismo ideológico y la diversidad de enfoques de los materiales didácticos que decida emplear no son discutibles), lo mejor que se puede hacer para neutralizar este cuestionamiento es lograr que la nueva materia, lejos de convertirse en un renovado intento de adoctrinamiento, sea capaz de generar en los alumnos justamente la capacidad crítica para evitarlo, al tiempo que profundiza en la dimensión ética personal.

Por otro lado, desde sectores ultraconfesionales se ha intentado desacreditar esta propuesta educativa, considerándola una especie de catequesis laica respecto a la cual se ha suprimido incluso la capacidad de elección de los padres, a quienes se usurpa el derecho a escoger para sus hijos una formación moral conforme a sus propios principios. Por insólito que parezca, se proclama que la educación para la ciudadanía es un ataque más a la familia y se insta a los progenitores a no permitir que sus hijos sean educados desde la apología del relativismo moral. Se trata de un llamamiento a la insumisión y a la rebelión ciudadana en toda regla, frente a lo que es percibido como el intento de creación de una moral de Estado antitética a los valores religioso-morales defendidos por un importante sector de la población. Curiosamente, quienes con mayor vehemencia se oponen a la formación escolar en valores democráticos son los mismos que defienden con igual firmeza la obligatoriedad de la enseñanza religiosa en la escuela (Jares, 2005). Sin embargo, la educación para la ciudadanía no debe ser vista como una alternativa y menos aún como un elemento antagónico a la enseñanza de la religión, sino que es una formación ética rigurosa que deben cursar con carácter obligatorio todos los estudiantes, al margen de la referencia religiosa, ya que todos ellos necesitan ser orientados adecuadamente en relación con las reglas que marcan la convivencia y el funcionamiento de las sociedades democráticas y el Estado de derecho.

\section{La educación para la ciudadanía pone a prueba las insuficiencias de la transversalidad}

En el marco del desarrollo reglamentario de la Ley Orgánica de Ordenación General del Sistema Educativo (LOGSE), las disposiciones legales que establecían los currículos de las distintas etapas educativas preveían la presencia de unos contenidos transversales referidos a una serie de ámbitos de aprendizaje de gran relevancia y demanda social, a la vez que un fuerte componente actitudinal, pero que no pertenecen al dominio de ninguna disciplina concreta, sino que precisan de la aportación

[4] También apuntados por Gómez Llorente (2005). 
conceptual de las distintas áreas o materias curriculares para su correcto tratamiento didáctico. Las enseñanzas transversales son, por lo tanto, aquellas dimensiones o temas recurrentes que, en vez de situarse en paralelo respecto a las áreas o disciplinas académicas que figuran en el currículo, las atraviesan o impregnan recíprocamente, originando una nueva perspectiva, un enfoque diferente de la realidad a través de grandes ejes de globalización e interdisciplinariedad.

Sin embargo, la tozuda realidad pone de manifiesto la persistencia de una organización de los contenidos escolares fuertemente disciplinar, lo que induce a una transversalidad dependiente y subsidiaria de la disciplinariedad. Ante un diseño curricular y una formación del profesorado -especialmente en la educación secundaria- firmemente asentados sobre los contenidos disciplinares, los temas transversales quedan en una situación de total desamparo frente al insalvable escollo de la lógica disciplinar. El resultado es que la transversalidad no ha cumplido los fines que perseguía y la propia propuesta ministerial así lo reconoce. La dispersión de la formación en valores a través de la transversalidad ha producido unos resultados claramente insuficientes. Por eso, a la vista de esta decepción, desde la Fundación Cives ${ }^{[5]}$ y en la misma línea adelantada por Peces-Barba se considera que la Educación para la ciudadanía es una asignatura imprescindible.

Eso no significa que este espacio educativo no deba estar cubierto de forma más o menos implícita por todas las materias y que deje de ser responsabilidad de todo el profesorado. Una escuela fundamentada sobre un proyecto cívico exige que todas las disciplinas escolares tengan una dimensión cívica, tanto en sus contenidos como en sus métodos (Audigier, 1999). Más aún: que toda la escuela esté imbuida de valores cívicos es una condición necesaria, pero no suficiente para asegurar una satisfactoria educación para la ciudadanía; en realidad, formar a los nuevos ciudadanos exige un sólido proyecto social, que va más allá de los centros escolares para afectar a toda la sociedad (Bolívar, 1996). Sin embargo, dado que existen graves problemas de convivencia en nuestra sociedad (intolerancia, xenofobia, racismo, violencia, consumismo, desigualdad, discriminación...), es perfectamente justificable la incorporación al currículo de una materia concreta y específica que, reforzando y no sustituyendo el indiscutible tratamiento transversal, sea capaz de construir y transmitir una cultura social (Audigier, 1999), es decir, abordar de forma expresa la educación en una serie de valores asociados a una concepción democrática de la organización social y política cuyo referente sea la Constitución (Gómez Llorente, 2005; Jares, 2005).

\section{Pero no va a ser fácil que la educación para la ciudadanía se consolide como mate- ria escolar}

La sociedad se encuentra en un proceso de constante producción de nuevos saberes que, en caso de que los considere suficientemente valiosos y apropiados, trata de convertir en nuevos objetos de enseñanza. Para ello dispone de tres alternativas:

[5] La Fundación Educativa y Asistencial Cives, constituida en 1996, trata de promover la ciudadanía plena en un Estado social y democrático de derecho, basando sus actuaciones en la filosofía del humanismo laico y en el ideal pedagógico de la Institución Libre de Enseñanza. La Fundación Cives y la Cátedra de Laicidad y Libertades Públicas Fernando de los Ríos del Instituto de Derechos Humanos Bartolomé de las Casas de la Universidad Carlos III han elaborado conjuntamente una propuesta sobre la nueva asignatura de Educación para la ciudadanía, que presentaron al Ministerio de Educación y Ciencia en 2005. 
incluirlos en una o varias de las asignaturas ya existentes, proponer enfoques comunes o transversales a todas ellas, o bien generar una nueva materia curricular (Audigier, 1999). Esta última es la decisión recientemente adoptada en nuestro país para la transmisión de valores cívicos, a sabiendas de que tal resolución iba a ser objeto de encrespadas controversias, con independencia del signo del gobierno proponente y del partido a quien correspondiese ejercer la oposición en ese momento. Las zafiedades del adoctrinamiento franquista, de no muy agradable recuerdo, planean aún sobre nuestras cabezas y sirven de hiriente arma arrojadiza contra los impulsores de la asignatura. Sea atribuible a una decisión valiente o desvergonzada, lo cierto es que el gato ya tiene puesto el cascabel, pero no será fácil que suene. Quiere esto decir que, por mucho que la educación para la ciudadanía haya sido en nuestro país una ausencia histórica que la LOE trata de enmendar (Martínez, 2005), los propósitos de formalizarla en una disciplina escolar "seria", con carácter de "materia principal, evaluable y explicada por profesores solventes y competentes", tal como reclamaba Peces-Barba en el artículo anteriormente mencionado, no van a tener una fácil materialización y su futuro parece bastante incierto.

En opinión de Robles (2005), "al margen del carácter intencional y propagandístico de asignatura semejante, tal educación no puede reglarse, institucionalizarse, ni canalizarse en un temario". Se dice que es una educación fuertemente ideologizada, politizada y enmarcada en un sistema doctrinal, en la que no caben especialistas ni tampoco asignaturas regladas. Quizás sea ésta la expresión más crispada de la confrontación mantenida entre el Ministerio de Educación y Ciencia y un sector del profesorado de Filosofía, en cuyo ámbito se percibe la nueva materia unas veces como una amenaza a su propia supervivencia y otras como un dominio exclusivo de su competencia, de donde se deriva, respectivamente, una actitud de rechazo o de reivindicación (Jares, 2005). Desde esta última posición, que pudiera considerarse gremialista o corporativista, se reclama para esta disciplina el nombre de Ética (o Ética cívica), como, en su opinión, corresponde a sus contenidos y finalidades. En este sentido, se insiste en que la mejor manera de abordar la educación para la ciudadanía es reforzar y extender a otros niveles y etapas del sistema educativo la actual asignatura de Ética, por ser la única que ha venido ofreciendo a los alumnos de educación secundaria una formación en valores con un enfoque no doctrinal ni confesional, al tiempo que se señala que los profesores de filosofía son los únicos profesionales que han recibido en su titulación una preparación específica para afrontar con garantía esos contenidos.

De manera análoga, pero desde el punto de vista de otro colectivo profesional, se piensa que una buena parte del contenido propio de la educación para la ciudadanía forma parte intrínseca de un currículo de ciencias sociales articulado en torno a problemas relevantes de nuestro mundo, cuya finalidad más destacada es precisamente la formación de los estudiantes para el desarrollo de un pensamiento social crítico y para el ejercicio de una ciudadanía democrática, con el objetivo de capacitarlos para la comprensión de la realidad en la que viven y para la intervención en ella con conocimiento de causa. Por lo tanto, sin negar la pertinencia de la presencia en la escuela de contenidos relacionados con la educación para la ciudadanía, sí se cuestiona la necesidad de hacerlo bajo el formato de una nueva asignatura, ya que sería suficiente con plantear una concepción de las ciencias sociales, no de una manera aditiva en un marco estrictamente disciplinar, sino ofreciendo una visión de la sociedad más integradora, a partir de cuestiones sociales de gran calado cuyo tratamiento didáctico exige la contribución de las diferentes disciplinas sociales. 
Como vemos, existen conocimientos escolares ya "asignaturizados" -Filosofía/Ética, Ciencias Sociales- que reclaman para sí el contenido de la educación para la ciudadanía y, cuando menos, ponen en duda la necesidad de introducir una nueva materia que recoja expresamente tales saberes cívicos. Y es que toda disciplina escolar es una construcción propia encargada de vehicular una cultura particular, que Forquin (1989) denomina "cultura escolar", y que está constituida por un detallado conjunto de conocimientos, competencias, actitudes y valores que la escuela, a petición de la sociedad, se encarga de transmitir explícita o implícitamente a los estudiantes como bagaje cultural y patrimonio común para todos los ciudadanos. En este sentido, cada una de las disciplinas escolares tiene como función aportar un contenido instructivo concreto y específico que se pone al servicio del objetivo que la sociedad asigna a las instituciones escolares: a saber, reintegrar unas generaciones de egresados dotadas de una serie de conocimientos, de competencias y de representaciones del mundo en el que viven, lo que hace de la escuela uno de los principales vehículos de transmisión intergeneracional (Audigier, 1997; Chervel, 1998).

En su momento y con gran fortuna, Chevallard (1985) se encargó de desarrollar y difundir el concepto de "transposición didáctica", con el que se refería al proceso de reelaboración del conocimiento científico disciplinar para convertirlo en conocimiento escolar; esta recomposición adaptativa supone la transformación del "saber experto" (procedente de la investigación) en "saber a enseñar" (propuesto en los programas, instrucciones oficiales y manuales) y, finalmente, en "saber enseñado" (enunciado en cada clase). Por el contrario, Chervel (1988) descarta completamente la idea de hacer descender los saberes enseñados desde la esfera del saber científico de referencia, reconociéndolos como creaciones originales de la escuela que, aun manteniendo ciertas relaciones con el saber erudito, han transformado profundamente el conocimiento científico hasta convertirlo en un saber específico objeto de enseñanza, aprendizaje y evaluación. De este modo, más que una simple suma de saberes pretendidamente científicos, las disciplinas escolares consisten en compartimentos estancos de saberes que organizan la distribución del tiempo escolar y la relación de los alumnos con el conocimiento, asumiendo funciones de aculturación de los estudiantes con el fin último de garantizar el cumplimiento de las finalidades atribuidas a la educación y a la escuela.

Todas las materias de enseñanza que contribuyen a configurar la cultura escolar constan de los siguientes ingredientes (Audigier, 1992, 1994, 1997; Chervel, 1988):

a) Un conjunto de conocimientos o contenidos explícitos compartidos por el profesorado y considerados como característicos de la disciplina, esto es, una "vulgata".

b) Una serie de ejercicios-tipo que contribuyen a definir y a delimitar la disciplina; si los contenidos expresos constituyen el eje central de la materia, los ejercicios resultan indispensables para su control, de manera que el éxito de una asignatura depende fundamentalmente de la calidad de los ejercicios susceptibles de ser propuestos.

c) En tercer lugar, unos procedimientos de motivación e incitación al estudio característicos, referidos no tanto a los medios que los profesores manejan en la cotidianeidad de la clase para despertar el interés de los alumnos, sino más bien a los esfuerzos constantes que la disciplina, como construcción global, tiene que llevar a cabo para justificar ante la sociedad (y, en particular, ante la propia colectividad 
escolar) su carácter esencial en la educación de niños y jóvenes, lo que determina el carácter ineludible de su presencia en la escuela. No olvidemos que ésta configura una especie de estadio en donde compiten todas las materias escolares, por lo que cada una de ellas debe estar constantemente en disposición de poder demostrar que su contribución a la formación de las nuevas generaciones es irreemplazable.

d) Por último, un aparato docimológico o conjunto de prácticas de evaluación, elemento decisivo de la disciplina para acreditar los aprendizajes realizados.

La escuela sólo puede enseñar saberes "escolarizables", es decir, aquellos conocimientos que están de acuerdo con el funcionamiento de la cultura escolar: transmisión de una vulgata, ejercicios comunes más o menos vehiculados o sugeridos por los manuales y por las tradiciones disciplinares, competencias evaluables según las modalidades institucionales... (Audigier, Crémieux y Mousseau, 1993). ¿Existe una cultura cívica escolar plenamente compartida por el profesorado y debidamente sistematizada para su transmisión a los estudiantes? ¿Con qué ejercicios se reforzará la vulgata y se asegurará el control de una materia sobre educación para la ciudadanía? ¿Qué procedimientos de motivación se pueden manejar para defender la necesidad de implantar esta asignatura en la escuela? ¿Se han previsto las modalidades y los instrumentos de evaluación para valorar el progreso de los alumnos? Sólo cuando estemos en condiciones de resolver satisfactoriamente estos interrogantes podremos asegurar que la educación para la ciudadanía se ha consolidado como un saber escolarizable, aceptado y estable.

Al sistema educativo le corresponde un papel determinante en la promoción de una ciudadanía activa tan necesaria para nuestros jóvenes. Ahora bien, es notorio que los expertos no han llegado todavía a un acuerdo compartido en relación con los contenidos propios de una materia de esta naturaleza, si bien parece suficientemente claro que los temas referentes a la democracia, la cohesión social y la participación ciudadana en ningún caso deben estar ausentes. En este sentido, es preciso un ajustado diseño basado en el consenso entre todas las fuerzas políticas y sociales sobre el tipo de ciudadano que se pretende modelar, el papel que se asigna a la educación formal en el desempeño de ese cometido y los contenidos que se consideran relevantes para lograrlo. Sin ello, la educación para la ciudadanía no sólo corre el riesgo de desdibujarse, sino que es posible que se acabe por banalizar o incluso desacreditar el propio significado de lo que debe ser una ciudadanía democrática. Y, desde luego, nada será posible si se prescinde de una adecuada formación específica del profesorado, sin menoscabo de la implicación efectiva de los responsables de las demás asignaturas, de la comunidad escolar y de la sociedad en su conjunto.

\section{Bibliografía}

Audigier, F. (1992). Pensar la geografia escolar. Un repte per a la didàctica. Documents d'Anàlisi Geogràfica, 21, 15-33.

Audigier, F. (1994). La didactique de la Géographie. En P. Desplanques (coord.), La Géographie en collège et en lycée (pp. 102-127). París: Hachette.

Audigier, F. (1997). Histoire et géographie: un modèle disciplinaire pour penser l'identité professionnelle. Recherche et Formation, 25, 9-21.

Audigier, F. (1999). L'éducation à la citoyenneté. Synthèse et mise en débat. París: INRP. 
Audigier, F.; Cremieux, C., y Mousseau, M.-J. (1993). Histoire et Géographie. En J. Colomb (dir.), Les enseignements en Troisième et Seconde. Ruptures et continuités (pp. 115-137). París: INRP.

Bolívar, A. (1996). Non scholae sed vitae discimus: límites y problemas de la transversalidad. Revista de Educación, 309, 23-65.

Chervel, A. (1988). L'histoire des disciplines scolaires. Réflexions sur un domaine de recherche. Histoire de l'Éducation, 38, 59-119. [Trad. cast.: Historia de las disciplinas escolares. Reflexiones sobre un campo de investigación. Revista de Educación, 295, 1991, 59-111].

Chervel, A. (1998). La culture scolaire. Une approche historique. París: Belin.

Chevallard, Y. (1985). La transposition didactique. Du savoir savant au savoir enseigné. Grenoble: La Pensée Sauvage (ed. de 1991). [Trad. cast.: La transposición didáctica. Del saber sabio al saber enseñado. Buenos Aires: Aique, 2000].

Eurydice (2005). La educación para la ciudadanía en el contexto escolar europeo. Bruselas: Eurydice.

Forquin, J.-C. (1989). École et culture: le point de vue des sociologues britanniques. Bruselas: De Boeck-Wesmael.

Gómez Llorente, L. (2005). La educación para la ciudadanía. Escuela Española, $3.665,12 / 05 / 2005$.

Jares, X. R. (2005). El lugar de la educación para la ciudadanía. Reflexiones y propuestas. Cuadernos de Pedagogía, 350, 88-92.

Marco Stiefel, B. (coord.) (2002). Educación para la ciudadanía. Un enfoque basado en el desarrollo de competencias transversales. Madrid: Narcea.

Martínez Rodríguez, J. B. (2005). Educacion para la ciudadanía. Madrid: Morata.

Peces-Barba, G. (2004): La educación en valores, una asignatura imprescindible. El País, 22/11/2004.

Robles López, J. (2005). Educación para la ciudadanía: Protágoras y Gorgias. El Catoblepas. Revista crítica del presente, 36.

http://www.nodulo.org/ec/2005/n036p10.htm

\section{Documentación}

Asociación Internacional para la Evaluación del Rendimiento Educativo (IEA): Civic Education Study. http://www.wam.umd.edu/ iea/>

Consejo de Europa: Education for Democratic Citizenship and Human Rights. http://www.coe.int/t/dg4/education/edc/default_EN.asp

Consejo de Europa. Comité de Ministros: Declaration and programme on education for democratic citizenship, based on the rights and responsibilities (Adopted by the Committee of Ministers on 7 May 1999, at its 104th Session). https://wcd.coe.int/ViewDoc.jsp?id=448125\&BackColorlnternet=9999CC\&Back ColorIntranet=FFBB55\&BackColorLogged=FFAC75

Consejo de Europa. Comité de Ministros: Recommendation Rec (2002)12 of the Committee of Ministers to member states on education for democratic citizenship (Adopted by the Committee of Ministers on 16 October 2002 at the 812th meeting of the Ministers' Deputies). 
https://wcd.coe.int/ViewDoc.jsp?id=313139\&BackColorlnternet=9999CC\&Back Colorlntranet=FFBB55\&BackColorLogged=FFAC75

Consejo de Europa. Ad Hoc Committee of Experts for the European Year of Citizenship through Education (CAHCIT): Education for Democratic Citizenship and Human Rights. Programme of Activities (2006-2009) "Learning and living democracy for all".

http://www.coe.int/t/dg4/education/edc/Source/Pdf/Documents/2006_5_ProgActi vities2006_2009.PDF

Ley Orgánica 2/2006, de 3 de mayo, de Educación (BOE núm. 106, de 4 de mayo). http://www.mec.es/mecd/gabipren/documentos/A17158-17207.pdf

Martín Cortés, I.: Una propuesta para la enseñanza de la ciudadanía democrática en España. Estudios de Progreso. Fundación Alternativas. http://www.falternativas.org/base/download/5c6c_20-09-06_EP\%2022.2006.pdf

Organización de las Naciones Unidas para la Educación, la Ciencia y la Cultura (UNESCO): Civics education for the twenty-first century.

http://portal.unesco.org/education/en/ev.phpURL_ID=4611\&URL_DO=DO_TOPI C\&URL_SECTION=201.html

Unión Europea: Ciudadanía de la Unión.

http://europa.eu/scadplus/leg/es/s18000.htm

Unión Europea: The future of education and citizenship policies: The Commission adopts guidelines for future programmes after 2006 (Press Releases, IP/04/315, 09/03/04).

http://europa.eu/rapid/pressReleasesAction.do? reference=IP/04/315\&format=HT ML\&aged=1 \&language $=E N \&$ guiLanguage $=$ en

Unión Europea. Comisión Europea: Europe for Citizens Programme 2007-2013. http://ec.europa.eu/citizenship/index_en.html

Unión Europea. Comisión Europea: Learning for active citizenship: a significant chaIlenge in building a Europe of knowledge. http://ec.europa.eu/education/archive/citizen/citiz_en.html

Unión Europea. Comisión Europea: Working Group "Open learning environment, active citizenship and social inclusion". Implementation of "Education \& Training 2010". Work Programme. Progress Report (November 2003).

http://ec.europa.eu/education/policies/2010/doc/opening-up-learning-and-citizenship_en.pdf 